\title{
Transformación digital de los departamentos de relaciones públicas y comunicación de una muestra de empresas españolas
}

\author{
Digital transformation of public relations and communication \\ departments of a sample of spanish companies
}

\author{
Cuenca-Fontbona, J., Matilla, K. y Compte-Pujol, M. ${ }^{1}$ \\ Recibido: 3-09-2019 - Aceptado: 4-02-2020 \\ DOI: https://doi.org/10.26441/RC19.1-2020-A5
}

\begin{abstract}
RESUMEN: El objetivo de este artículo es analizar el grado de madurez en la transformación digital de los departamentos de relaciones públicas y comunicación de las empresas de mayor facturación españolas asociadas a la Asociación de Directivos de Comunicación-Dircom, mediante el modelo de transformación digital de Westerman, Bonnet y McAfee $(2012,2014)$, basado en los vectores de liderazgo y capacidad digital. Para este cometido se cursó un cuestionario a sus máximos responsables de comunicación.
\end{abstract}

Los resultados identifican que el $73,6 \%$ de los departamentos de comunicación de las empresas participantes están en un momento avanzado de esta transformación, condicionando cambios culturales al gobierno corporativo, la contratación de nuevos perfiles, la reorganización del trabajo y a focalizar las áreas donde cabe una mejora que maximice el retorno de los recursos invertidos. El $85 \%$ de estos departamentos tienen una visión integral de la comunicación digital, aunque reconocen que aún deben automatizar muchos de sus procesos.

Palabras clave: transformación digital; comunicación digital; tecnologías de la información; relaciones públicas; departamentos de comunicación; empresas españolas.

\begin{abstract}
The goal of this article is to analyze the degree of maturity in the digital transformation of the public relations and communication departments of the companies with the highest turnover that belong to the Spanish Association of Communication Directors-Dircom by applying the model for digital transformation proposed by Westerman, Bonnet and McAfee $(2012,2014)$, based on the vectors of leadership and digital capacity. For this purpose, a questionnaire was sent to those responsible for communication of this type of companies.
\end{abstract}

Our results identify that $73.6 \%$ of the communication departments are at an advanced stage of this

\footnotetext{
${ }^{1}$ Joan Cuenca-Fontbona es Doctor en Comunicación y Humanidades y Profesor Investigador de la Facultad de Comunicación y Relaciones Internacionales Blanquerna de la Universidad Ramon Llull de Barcelona. Imparte asignaturas de Teoría, técnicas y estrategia en relaciones públicas, Comunicación corporativa, Introducción a la publicidad, Relaciones públicas y el marketing, etc. Es Director del Máster en Estrategia y Dirección de Relaciones Públicas y Comunicación y miembro del Grupo de Investigación en Estrategia y Creatividad en Publicidad y Relaciones Públicas. joancf@blanquerna.url.edu, http://orcid.org/0000-0001-5807-9442

Kathy Matilla es Doctora especializada en relaciones públicas, Profesora Investigadora de Planificación estratégica de las relaciones públicas y Comunicación corporativa, y miembro del Grupo de Investigación en Estrategia y Creatividad en Publicidad y Relaciones Públicas de la Facultad de Comunicación y Relaciones Internacionales Blanquerna, de la Universidad Ramon Llull de Barcelona. kathyms@blanquerna.url.edu, http://orcid.org/0000-0001-5247-3289.

Marc Compte-Pujol es Doctor en Estudios Avanzados en Comunicación por la Universidad Ramón Llull de Barcelona y miembro del grupo de investigación interuniversitario Learning, Media \& Social Interactions (LMI). Es profesor de Publicidad, Relaciones públicas y Audiovisuales en la Facultad de Empresa y Comunicación de la Universidad de Vic-Central de Cataluña y en la Facultad de Comunicación y Relaciones Internacionales Blanquerna de la Universidad Ramón Llull. marc.compte@uvic.cat, https://orcid.org/0000-0002-6694-2485
} 
transformation, conditioning cultural changes to corporate governance, the demand for new profiles, the reorganization of work, and focusing on areas where there is an improvement that maximizes the return of resources invested. $85 \%$ of these departments have an integral vision of digital communication, although they recognize that they must automate many of their processes.

Keywords: digital transformation; digital communication; information technology; public relations; communication departments; spanish corporations.

\section{Introducción}

En las últimas cuatro décadas lo que ha revolucionado a la historia de la humanidad, por la influencia, magnitud y rapidez de su implementación, han sido las Tecnologías de la Información y la Comunicación (TIC), que han dado forma a lo que hoy designamos como Revolución Digital (Linares, 2018).

La digitalización, definida por la $\mathrm{OCDE}^{2}$ como una tecnología de utilidad general que da soporte a todos los sectores productivos (Berges, 2018), ha convergido en la denominada nueva economía. Un nuevo escenario global, intensamente interconectado, que da importancia a las ideas, a la información y a las relaciones (Kelly, 1997). En los últimos años este contexto ha transformado la práctica totalidad de todo tipo de procesos en todos los ámbitos de actividad de las economías más avanzadas. Una transformación analizada por múltiples estudios internacionales como el Digital Economy and Society Index, elaborado por la Comisión Europea (DESI, 2019), el informe Global Connectivity Index GCI 2017 de Huawei, el The Global Information Technology Report, publicado por el Foro Económico Mundial (Baller, Dutta y Lanvin, 2016), la investigación de Constellation Research (Sato y Wang, 2019), el estudio The Year of Digital Decisions de DXC Technology (Smith y Davies, 2019), el i-scoop.eu de 2018 y la base de datos actualizada de la Comisión de la Unión Europea, la Agenda Digital para Europa (Maciejewski y Gouardères, 2018).

España, desde el punto de vista del uso de la tecnología a nivel empresarial, ocupa la posición 45 en el ranking mundial y la $8^{\mathrm{a}}$ posición europea y, según el indicador agregado (indicador de la Economía y la Sociedad Digital), que consolida cinco dimensiones fundamentales -conectividad, capital humano, uso de Internet, integración de tecnología digital y servicios públicos digitales-, España se sitúa en el $15^{\circ}$ puesto entre los 28 estados miembros de la Unión Europea (Berges, 2018): una posición discreta a nivel internacional.

El informe íncipy-inesdi revela que la mayoría de las empresas reconocen que aún no destinan una parte importante de su presupuesto total a la transformación digital y que incluso más de la mitad de las empresas, aunque conscientes, no disponen tan siquiera de una hoja de ruta digital formalizada (Sánchez, 2017). La razón que explicaría, en parte, esta situación, la encontramos en el Informe de Minsait 2018, cuando detalla que España es uno de los países que no fue capaz de extraer todo el partido posible a la revolución tecnológica hasta el 2015, fecha a partir de la cual esta situación parece haberse revertido (Beldarrain, 2018). En estos momentos, según el Estudio de Indicadores Digitales en la empresa española de IDC Research España (2018), el 75\% de las empresas españolas han emprendido ya su transformación digital.

Esta información contrasta con los resultados del informe Global Connectivity Index GCI 2017, que especifica que, aunque España no se incluye en el grupo de países frontrunners liderados por Estados Unidos, Singapur y Suecia, sí ostenta un lugar de liderazgo puesto que, en estos momentos, está encabezando el grupo de países que el informe define como adopters, es decir, países que son conscientes y así están dispuestos a usar la tecnología e invertir recursos en ella porque entienden

\footnotetext{
${ }^{2}$ La OCDE es la Organización para la Cooperación y Desarrollo Económico, http://www.oecd.org/.
} 
que es beneficiosa para su desarrollo. Además, el Barómetro sobre la Madurez Digital en España 2018 (Crespo y Pariente, 2018), certifica que la mayoría de las grandes corporaciones ya han incorporado la transformación digital en todas sus áreas funcionales.

La aplicación de las nuevas tecnologías a la organización, el Internet de las Cosas (IoT), el Big Data, el Cloud, los Wearables, las Contact lens smartphones, la TV4K, los teléfonos móviles 5G, el Blockchain, la realidad virtual y aumentada, la inteligencia artificial, la robótica, los drones, los bitcoins, la geolocalización, la ciberseguridad, las impresoras 3D, el transporte 2.0 o las culturas derivadas como el movimiento Maker, etc., han provocado la aparición de nuevos perfiles profesionales o la exigencia de incorporar nuevas habilidades a las profesiones tradicionales (Alexander, Stefanova y Zahidi, 2018), y han propiciado crear nuevos modelos de negocio, mejorar los existentes y cambiar el modo de relacionarse con los públicos estratégicos (Rodríguez y Castillo, 2017).

La expansión de estas tecnologías ha promovido escenarios en el que los modos de conexión y comunicación digitales impregnan todos los aspectos de nuestras vidas y las relaciones públicas y la comunicación no han sido ajenas a estos cambios y la literatura especializada revela que las organizaciones españolas, poco a poco, van descubriendo las ventajas que presenta el ágora virtual para gestionarlas. La multiplicación de las demandas de información, la distancia, la regionalización de la información, la velocidad de la transmisión, la convergencia de diferentes tecnologías y la necesidad y capacidad de reaccionar rápidamente -especialmente en situaciones de crisis- han llevado a los directivos del área a perfeccionar las técnicas de actuación mediante estos sistemas interactivos multimedia.

La consultora global de comunicación y asuntos públicos LLYC afirma que liderar la transformación digital es uno de los principales retos a los que se enfrentan los profesionales de la comunicación (prNoticias, noviembre de 2017). Este entorno disruptivo no ha alterado la necesidad de estos profesionales de compartir sus ideas en el marco de una alocución, pero ha modificado completamente las secuencias de su trabajo, decuplicando las posibilidades de investigación, producción y difusión (González Molina, 2011), pero, en estos momentos, aun siendo un desafío imprescindible, la transformación digital de los departamentos de relaciones públicas y comunicación se está desplegando de forma desigual (Zerfass, Verhoeven, Moreno, Tench y Verčič, 2016).

Ante este contexto digital, una mejor dirección y gestión estratégicas de la comunicación está exigiendo un comportamiento diferente de quien la lidera y de una capacitación en nuevas tecnologías y social media, del conocimiento de la medición, del análisis de datos y del big data derivados de este nuevo universo relacional, y de brindar mejor valor a los stakeholders de las organizaciones con nuevas formas de gestionar la actividad de las relaciones públicas y la comunicación. Una transformación digital que no consiste únicamente en la tecnología que se utiliza, sino que implica la inversión en el desarrollo de capacidades digitales que deben de estar alineadas con la estrategia corporativa (Lorenzo Ochoa, 2016).

La hipótesis que plantea esta investigación es que la digitalización de los departamentos de relaciones públicas y comunicación de las organizaciones españolas es elevada. Así, el propósito principal de este trabajo es el de realizar una exploración, mediante la consulta y análisis de fuentes especializadas y sectoriales y el envío de un cuestionario online a una muestra de calidad de directivos funcionales de relaciones públicas y comunicación, para contribuir al enriquecimiento del estado de la cuestión acerca del grado de madurez en la transformación digital de los departamentos de relaciones públicas y comunicación de las empresas españolas de mayor facturación asociadas a Dircom y que permita, además, corroborar esta hipótesis.

Como objetivos secundarios, se persigue conocer: (a) el nivel de liderazgo necesario para impulsar la transformación digital de los departamentos de relaciones públicas y comunicación de la muestra 
participante y, (b) los recursos tecnológicos en las que estos departamentos invierten para cambiar su gestión operativa y aumentar su eficiencia y su competitividad.

\section{Marco referencial}

Actualmente no existe una definición consensuada acerca de lo que significa la transformación digital (Schallmo y Williams, 2017; Teichert, 2019), ya que el concepto presenta diferentes enfoques según sean el director funcional, la organización o el sector económico (Lorenzo Ochoa, 2016). De todas formas, en general se concibe la transformación digital como el proceso de gestión que orienta la cultura, la estrategia, las metodologías y las capacidades de una organización a partir de las tecnologías digitales (Crespo y Pariente, 2018) que, por un lado, ha permitido el surgimiento de empresas unicornios (Lee, 2013), capaces de facturar millones de euros en pocos años y, por otro lado, ha estimulado la reinvención de modelos de negocio más tradicionales y ha abarcado, según la consultora McKinsey\&Company (2017), a todas las áreas funcionales de la organización.

La transformación digital se inicia con una manera distinta de pensar (Linares, 2018; Sánchez, 2015) que demanda un cambio importante en la cultura, la estructura y las operaciones de las organizaciones. Esta actitud digital (Aced, Arqués, Benítez, Llodrà y Sanagustín, 2009; Aced, 2010) también debe darse en los departamentos de relaciones públicas y comunicación, ya que la consolidación de Internet como herramienta de comunicación y de intercambio de información y el constante uso de las redes sociales han modificado los modelos de trabajo del área comunicativa (Cook, 2019; Cuenca-Fontbona, Matilla y Compte-Pujol, 2016; González-Canomanuel, 2018; Whatmough, 2018; Zerfass, Tench, Verčič, Verhoeven y Moreno, 2014) y la nueva realidad dominante facilita una comunicación inmediata, instantánea y directa (Wilcox, Cameron y Reber, 2015).

Invertir en este tipo de actitud profesional redefine la relación con los clientes, con los proveedores, con los propios empleados y, en definitiva, con todo el ecosistema empresarial (Belzunce, Toledo y Fernández, 2018; Berges, 2018) y puede producir unas relaciones de mejor calidad y una reputación corporativa más ventajosa (Cuenca-Fontbona, Matilla y Compte-Pujol, 2016).

La Public Relations Consultant's Association y el Chartered Institute of Public Relations ya apreciaron algunos indicios de la transición digital en las relaciones públicas, y constituyeron en 1999 la Internet Commission para analizar la incursión de la digitalización en este ámbito profesional (Gordon, 2011). Un estudio financiado por esta comisión concluía que la interactividad que proporciona Internet es especialmente relevante para las relaciones públicas porque estimula el diálogo, es decir, la comunicación simétrica bidireccional propugnada por James Grunig en 1984, logrando una mejora en las relaciones con los públicos gracias al mejor uso, por ejemplo, del sitio web de una organización (Len-Ríos y Cameron, 2001; Castillo-Esparcia y Smolak, 2017).

Para comprender este nuevo entorno disruptivo y su desigual desarrollo aplicativo, se han desarrollado diversos modelos para analizar esta transición. Uno de los modelos pioneros es el de los profesores del Center for Digital Business del MIT (Massachusetts Institute of Technology), George Westerman, Didier Bonnet y Andrew McAfee quienes realizaron una investigación, conjuntamente con Capgemini Consulting, en el que participaron 400 empresas norteamericanas cotizadas y en la que estudiaron las iniciativas y oportunidades digitales que estaban siendo aprovechadas por estas empresas. Los resultados fueron publicados en la revista MIT Sloan Management (2012) conforme existe una correlación clara entre las empresas digitalmente maduras y aquellas que son más competitivas y poseen un potente rendimiento financiero. De este estudio concibieron el concepto de madurez digital que describe cómo diferentes empresas están reac- 
cionando o actuando a las oportunidades digitales, y diseñaron un modelo pionero basado en el Indicador de Madurez Digital (IMD).

El término "madurez" se refiere a un estado de ser completo, perfecto o listo, y es el resultado del progreso en el desarrollo de un sistema. Así pues, el término "madurez digital" refleja específicamente el estado de la transformación digital de una empresa, es decir, describe lo que una empresa ya ha logrado gracias a los esfuerzos de transformación y cómo ésta se prepara sistemáticamente para adaptarse competitivamente a un entorno cada vez más digital (Teichert, 2019)

El indicador de Madurez Digital (IMD) se mide a partir de la combinación de dos dimensiones diferentes que están relacionadas: (a) el liderazgo (con visión digital) para impulsar la transformación digital, es decir, la intensidad de la gestión transformacional o el empuje, la convicción y la creencia por parte del gobierno de la organización en una firme apuesta de valor diferencial y beneficiosa para el consumidor, y (b) la capacidad digital, que hace referencia a la intensidad de las iniciativas tecnológicas en las que una organización invierte para cambiar su gestión operativa y aumentar su competitividad (Westerman, Bonnet y McAfee, 2012, 2014).

De acuerdo con este modelo, las empresas pueden tener cuatro posibles niveles de madurez digital: alta intensidad digital y gestión de la transformación, baja intensidad digital de gestión de la transformación, o una mezcla de alta y baja para las dos dimensiones:

Nivel 1 - Beginners o principiantes digitales que han experimentado e implantado soluciones tecnológicas y aunque algunas de ellas aportan valor al negocio, la mayoría no lo hace. Les falta una estrategia coordinada y visión de transformación integral.

Nivel 2 - Fashionistas o seguidoras de la moda digital, fuertemente motivadas para provocar el cambio digital, pero con una estrategia que no está basada en un conocimiento real de cómo esta transformación puede y debe aportar valor al negocio.

Nivel 3 - Conservatives o conservadoras digitales que entienden la importancia de la estrategia, de la coordinación, de las cuestiones de gobernanza y de la cultura organizacional a la hora de abordar un proceso de transformación, pero que son muy escépticas sobre el valor de lo digital en estos procesos. Aunque dispuestas a invertir en cambio digital, su lentitud les hace perder oportunidades en detrimento de otras organizaciones más arriesgadas.

Nivel 4 - Digital Masters, digitari o transformadas que saben cómo aportar valor al negocio desde la transformación digital. Combinan visión y gobernanza con el compromiso por la inversión. Gracias a la visión global y a la integración de toda la organización en la estrategia de transformación digital logran desarrollar una cultura digital que les permite incorporar nuevos cambios a su modelo de negocio.

\section{Metodología}

En primer lugar, se revisó la literatura especializada para conocer los diferentes modelos de madurez digital existentes, identificándose 22 modelos (Teichert, 2019). Se eligió el modelo sobre madurez digital elaborado por G. Westerman, D. Bonnet y A. McAfee $(2012,2014)$, tras obtener previamente la aprobación expresa de los autores para su utilización, por ser un modelo de traza general que facilita su adaptación a cualquier industria, área o departamento (Lorenzo Ochoa, 2016) y porque es uno de los modelos pioneros que en 2012 ya ofrecía un marco integrado que permitía a cualquier tipo de organización evolucionar progresivamente en el desarrollo de las capacidades claves para ser exitosas en la nueva era digital. Es uno de los referentes de autores, profesionales y 
académicos que, posteriormente, han desarrollado y aplicado diferentes modelos de madurez digital a diferentes campos de actuación (Teichert, 2019).

Acto seguido, se elaboró un cuestionario online diseñado con el servicio Google Drive, formularios de Google y consistente con el estudio sobre madurez digital de estos autores. El cuestionario se redactó en castellano y constó de 2 secciones que perseguían verificar o refutar la hipótesis de partida. En la primera de las secciones se preguntó acerca de las capacidades de liderazgo del departamento de relaciones públicas y comunicación. La segunda sección trató sobre las capacidades digitales del departamento.

Ambas secciones contienen diez preguntas factuales cada una, en cuyas opciones de respuesta se ha establecido, siguiendo el modelo original, una escala Likert de siete niveles. El formato de respuesta va de "totalmente en desacuerdo", con una valoración ordinal de 1, a "totalmente de acuerdo", con una valoración ordinal de 7. Para este estudio se ha utilizado la posibilidad sumativa de este tipo de escala. Así pues, para presentar los resultados, se han sumado razonablemente el grupo de elementos de cada una de las dos partes opuestas de la escala, considerando a las puntuaciones 1, 2 y 3 (diferentes grados de desacuerdo) como desacuerdo con la cuestión planteada y, a las 5, 6, y 7 (diferentes grados de acuerdo) como de acuerdo con la cuestión planteada. La puntuación 4 (ni en desacuerdo ni en acuerdo) se ha considerado neutra.

De estas dos secciones se obtiene como resultado una puntuación de entre 10 y 70 puntos. Según el modelo de Westerman, Bonnet y McAfee $(2012,2014)$, en función de la puntuación final se etiqueta a los departamentos de relaciones públicas y comunicación según los cuatro niveles de madurez digital identificados por su modelo de estudio: los beginners, los fashionistas, los conservatives y los digital masters.

Estos cuatro niveles de madurez digital se representan gráficamente en una matriz de doble entrada, donde el eje de abscisas expresa la variable capacidad de liderazgo, y el eje de ordenadas la variable capacidad digital del departamento. El modelo considera una capacidad baja, en ambas variables, una puntuación de entre 10 y 41, y una capacidad alta, en ambas variables también, en una puntuación entre 42 y 70.

Así pues, como se puede observar en la Figura 1, cuando el liderazgo digital del departamento es bajo (puntuación entre 10 y 41) este departamento se situaría a la izquierda de la matriz (beginners o bien fashionistas). Cuando el liderazgo digital es elevado (puntuación entre 42 y 70) el departamento se encontraría a la derecha de la matriz (conservatives o bien digital masters). Y, cuando las capacidades digitales son bajas (puntuación entre 10 y 41) el departamento se situaría en la parte baja de la matriz (beginners o bien conservatives). Finalmente, cuando las capacidades digitales son elevadas (puntuación entre 42 y 70) el departamento se ubicaría en la parte alta de la matriz (fashionistas o bien digital masters). La posición resultante permitirá llegar a conclusiones respecto al estado de la cuestión sobre la madurez digital de los departamentos de relaciones públicas y comunicación estudiados. 
Figura 1. Four levels of Digital Mastery

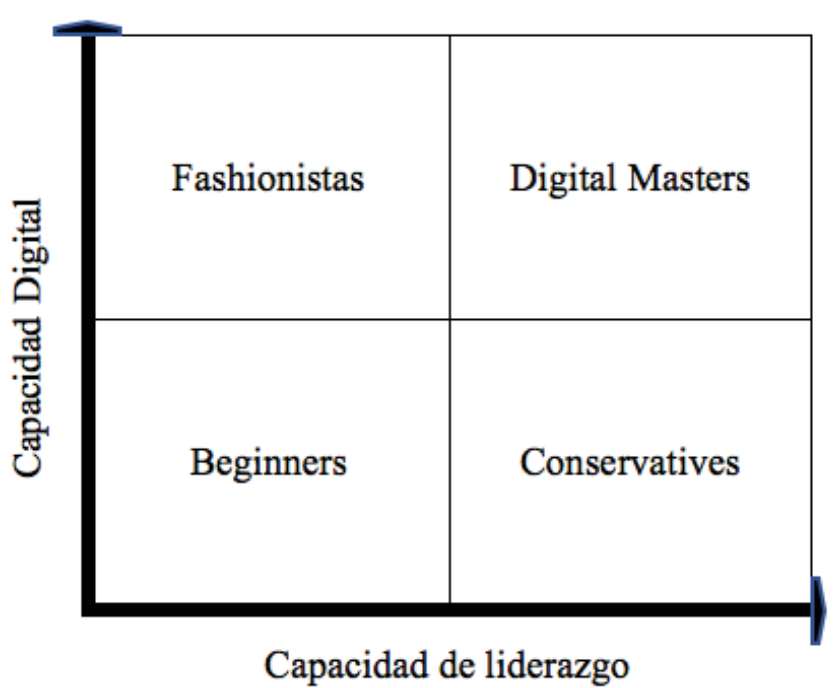

Fuente: Westerman, Bonnet y McAfee, 2012, 2014. Elaboración propia.

El cuestionario autoadministrado se envió por correo electrónico, en el marco de los métodos de administración a distancia, con una presentación y una breve descripción del propósito del estudio e incluyendo el enlace que daba acceso al cuestionario en Google Drive, en dos oleadas semanales consecutivas desarrolladas entre el 12 y el 25 de noviembre de 2018, a las direcciones electrónicas de los 1.067 directivos funcionales de relaciones públicas y comunicación de las empresas españolas de mayor facturación que son socios de la Asociación de Directores de Comunicación-Dircom ${ }^{3}$ y que, por su alto nivel de representatividad, constituyen una muestra de calidad. La información de contacto de esta muestra se obtuvo del directorio Dircom $2017^{4}$ y las direcciones electrónicas se obtuvieron, una a una, de diversas fuentes y artesanalmente.

Finalmente, del universo de las 1.067 empresas contactadas, se consiguió la respuesta, durante el periodo anunciado, de 125 empresas $(11,71 \%)$ que conformaron la muestra de análisis.

El grueso de las empresas de la muestra está localizado en Barcelona (37,5\%) y en Madrid (28,8\%) y, el resto, en diferentes capitales y poblaciones españolas como Cádiz, Granada, Palma de Mallorca, Valencia, Valladolid, Zaragoza, etc. $(33,7 \%)$. El directorio Dircom clasifica a estas empresas en 10 categorías sectoriales. Las respuestas que se han recibido pertenecen a organizaciones de la categoría de Servicios y Ocio (22,4\%), seguidas por la de Alimentación, Bebidas y Tabaco $(21,6 \%)$, Comercio y Distribución (18,4\%), Automoción, Transporte, Autopistas y Aparcamientos (13,6\%), Entidades Financieras, Bancos, Cajas y Gestoras y Telecomunicaciones, Informática e Internet (6,4\%), Construcción, Siderometalúrgica, Energía y Electricidad (4\%), Auditorias, Consultoras y Bufetes de Abogados (3,2\%), Sector Químico y Farmacéutico (2,4\%) y Seguros (1,6\%). Al final se presentan los resultados de cada una de las cuestiones planteadas y se consigna el cómputo total de las 125 empresas participantes que han contestado todas y cada una de las cuestiones planteadas.

\footnotetext{
${ }^{3}$ La asociación Dircom es una asociación profesional que agrupa a los directivos ya los profesionales de la comunicación de las empresas, instituciones y consultoras españolas. Nace en 1992 de la iniciativa de un grupo de destacados profesionales de la comunicación motivados por la creciente importancia y alcance de sus responsabilidades en la empresa y la sociedad. Dircom tiene como visión poner en valor la función de la comunicación y del director de comunicación en las organizaciones, de tal forma que dicha competencia y sus responsables sean considerados co mo un área y un directivo estratégicos. Su misión la lleva a cabo a través de cuatro ejes estratégicos: reconocimiento, desarrollo profesional, networking y gestión responsable/RSC. http://www.dircom.org/sobre-dircom/que-es-dircom

${ }^{4}$ Directorio Dircom 2017. Anuario de la Comunicación 2017. Almería: Asociación de la Prensa de Almería - FAPE. http://almeria.fape.es/wp-content/uploads/2017/07/DIRCOM_Directorio_2017.pdf
} 
En este punto cabe aclarar que, aunque en nuestra investigación denominamos a las áreas funcionales objeto de estudio como departamentos de relaciones públicas y comunicación, se constata que existe una absoluta carencia de homogeneización en la denominación del departamento, tal y como confirma el estudio de Matilla, Miranda, Compte-Pujol y Oliveira (2018) acerca de la gran divergencia en la denominación de los departamentos de comunicación en España. Así pues, los participantes en realidad se autodenominan genérica y prevalentemente como directores de comunicación (dircoms) y, consecuentemente, son los máximos responsables de los departamentos de comunicación (Cabrera-Cabrera y Almansa-Martínez, 2016).

El análisis estadístico de los datos se ha realizado a partir del abanico de resultados y gráficos que proporciona Google Drive, un sistema reconocido por la exactitud de sus cálculos y su versatilidad para visualizar resultados.

Como limitaciones del estudio se advierte que ni el universo seleccionado ni la muestra de análisis son representativos de la totalidad de las empresas españolas ni de la totalidad de los directores de comunicación que ejercen su responsabilidad funcional en el Estado español, universo que no ha sido posible identificar documentalmente. Además, la participación según categoría económica ha sido desigual y esto limita cualquier análisis comparativo o conclusión de tipo sectorial. Sin embargo, se trata de una muestra de alta calidad que permite considerar los resultados de la investigación como tendencias generales.

\section{Análisis de los resultados}

\subsection{Capacidades de liderazgo de los departamentos de Relaciones Públicas y Co- municación participantes}

Los resultados acerca del liderazgo digital se detallan a continuación en la Tabla 1 y en la Figura 2. En la $1^{\text {a }}$ columna se han consignado las preguntas formuladas:

Tabla 1. Capacidades de liderazgo del departamento de comunicación

\begin{tabular}{|c|c|c|c|c|c|c|c|}
\hline Cuestionario & $\%$ & $\%$ & $\%$ & $\%$ & $\%$ & $\%$ & $\%$ \\
\hline $\begin{array}{l}\text { Por favor responda a cada pregunta usando una escala de } 1 \text { a } 7(1 \\
=\text { totalmente en desacuerdo, } 4=\text { neutro, } 7=\text { totalmente de acuerdo) }\end{array}$ & 1 & 2 & 3 & 4 & 5 & 6 & 7 \\
\hline Estamos en un momento avanzado de la transformación digital & 0,8 & 1,6 & 6,4 & 17,6 & 16 & 24 & 33,6 \\
\hline $\begin{array}{l}\text { Los altos ejecutivos tienen una visión transformadora del futuro } \\
\text { digital de nuestra empresa. }\end{array}$ & 3,2 & 1,6 & 4 & 16 & 16,8 & 28 & 30,4 \\
\hline $\begin{array}{l}\text { Los altos ejecutivos funcionales y los miembros del departamento de } \\
\text { comunicación estamos alineados respecto a la transformación digital. }\end{array}$ & 1,6 & 0,8 & 3,2 & 11,2 & 16,8 & 34,4 & 32 \\
\hline $\begin{array}{l}\text { El departamento de comunicación está promoviendo los cambios } \\
\text { culturales necesarios para la transformación digital. }\end{array}$ & 0,8 & 1,6 & 4,8 & 9,6 & 13,6 & 38,4 & 31,2 \\
\hline $\begin{array}{l}\text { Los miembros del departamento de comunicación tienen la posi- } \\
\text { bilidad de participar en la transformación digital. }\end{array}$ & 0,8 & 0 & 1,6 & 9,6 & 20 & 30,4 & 37,6 \\
\hline $\begin{array}{l}\text { Las acciones de comunicación digital están coordinadas estraté- } \\
\text { gicamente con el resto de operaciones de comunicación. }\end{array}$ & 0 & 1,6 & 1,6 & 6,4 & 15,2 & 30,4 & 44,8 \\
\hline $\begin{array}{l}\text { Los roles y las responsabilidades para gestionar la actividad digi- } \\
\text { tal de comunicación están claramente definidas. }\end{array}$ & 1,6 & 1,6 & 8,8 & 8,8 & 23,2 & 23,2 & 32,8 \\
\hline $\begin{array}{l}\text { Las acciones de comunicación digital se evalúan mediante indi- } \\
\text { cadores de rendimiento clave. }\end{array}$ & 4,8 & 3,2 & 4,8 & 12 & 12,8 & 32,8 & 29,6 \\
\hline $\begin{array}{l}\text { Los responsables de tecnologías de la información y del departa- } \\
\text { mento de comunicación trabajamos conjuntamente. }\end{array}$ & 2,4 & 4 & 2,4 & 13,6 & 20,8 & 30,4 & 26,4 \\
\hline $\begin{array}{l}\text { El rendimiento de la unidad de tecnologías de la información sa- } \\
\text { tisface las necesidades del departamento de comunicación. }\end{array}$ & 3,2 & 5,6 & 3,2 & 16 & 25,6 & 31,2 & 15,2 \\
\hline Medias & 1,92 & 2,16 & 4,08 & 12,08 & 18,08 & 30,32 & 31,36 \\
\hline
\end{tabular}

Fuente: elaboración propia 
El 73,6\% de las empresas españolas de mayor facturación participantes, es decir, casi tres cuartas partes de la muestra analizada, explican que su departamento de comunicación se encuentra en un momento avanzado o muy avanzado en cuanto a la transformación digital se refiere. Y únicamente un $8,8 \%$ de estas organizaciones se sitúan en la zona que evidencia su escasa o nula evolución. El resto, el 17,6\%, no cree que la transición digital sea una cuestión relevante a destacar en su departamento.

La información anterior coincide, en gran medida, con la mayoritaria visión transformadora del futuro digital que tienen el $75,2 \%$ de los altos ejecutivos de las compañías participantes, y con el alineamiento del resto de ejecutivos funcionales y los miembros de los departamentos de comunicación que suman el 83,2\%. También coincide, grosso modo, la proporción de un $8,8 \%$ de los altos ejecutivos quienes tienen una baja visión transformadora del futuro digital por 5,6\% de los puestos funcionales.

Según el $88 \%$ de los máximos responsables de comunicación, los miembros del departamento de comunicación tienen la posibilidad de participar de la transformación digital de su compañía, y un $83,2 \%$ de estos departamentos están promoviendo los cambios culturales necesarios, en mayor o menor medida, para que la transformación digital se produzca.

La mayoría de los miembros de la muestra analizada, el 90,4\%, están de acuerdo en que las acciones de comunicación digital están coordinadas estratégicamente con el resto de operaciones de comunicación de la organización. Y, en cuanto a la responsabilidad de la función digital dentro de este departamento, el 79,2\% estaría de acuerdo en que los roles y las responsabilidades para gestionar la actividad digital de comunicación están claramente definidas. Aun así, se observan dos proporciones a tener en cuenta, el 13,3\% ve en mayor o menor medida difusa esta responsabilidad, y el $8,8 \%$ tampoco parecería tenerla claramente definida.

En otro orden temático, se observa que el 75,2\% de los responsables de los departamentos de relaciones públicas y comunicación participantes aseguran que las acciones de comunicación digital se evalúan mediante indicadores de rendimiento clave de los procesos diseñados de comunicación. En cambio, un $12,8 \%$ afirma que la monitorización es nula o casi nula. Un 12\% no contestan en ningún sentido.

En este mismo bloque el 77,6\% expresa diferentes grados de acuerdo conforme a que los responsables de tecnologías de la información y los responsables del departamento de comunicación estén, en estos momentos, trabajando conjuntamente. También el $72 \%$ de los encuestados creen que el área responsable de las tecnologías de la organización satisface los requerimientos del departamento de comunicación. Cabe destacar, de todas formas, que únicamente el 15,2\% de esta facción estaría muy de acuerdo acerca de esta comunión.

Estas dos últimas cuestiones relacionadas con la colaboración entre estas dos unidades operativas suman, aproximadamente, una cuarta parte de quienes piensan que la conexión no es del todo armónica, más quienes piensan que no es ni armónica ni discrepante, $22,4 \%$ y $28 \%$ respectivamente.

Al final de esta sección se obtiene una puntuación de entre 10 y 70 (Figura 2). En esta ocasión las organizaciones suman una puntuación media de 55,57, así pues, se observa que el liderazgo digital, según el modelo de transformación digital de Westerman, Bonnet y McAfee (2012) es importante, puesto que la puntuación se sitúa entre 42 y 70 .

Hay 111 organizaciones de las 125 de la muestra de las empresas españolas de mayor facturación estudiadas $(88,8 \%)$, que se reconocen motivadas, convencidas y ejerciendo una influencia real en el estilo de gestión de sus equipos para su adaptación digital.

El liderazgo digital de las empresas españolas participantes se sitúa a la derecha de la matriz entre los denominados conservatives y los digital masters, según ha quedado expuesto en la Figura 2. 
Figura 2. Capacidades de liderazgo del departamento de Relaciones Públicas y Comunicación

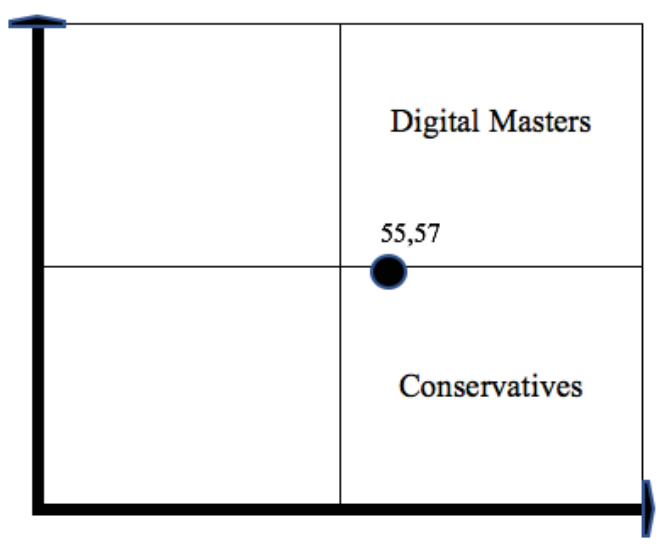

Liderazgo digital

Fuente: elaboración propia.

\subsection{Capacidades digitales del departamento de Relaciones Públicas y Comunica- ción}

Los resultados acerca de las capacidades digitales se detallan a continuación en la Tabla 2 y en la Figura 3. En la $1^{\text {a }}$ columna se han consignado las preguntas formuladas:

Tabla 2. Capacidades digitales del departamento de comunicación

\begin{tabular}{|c|c|c|c|c|c|c|c|}
\hline Cuestionario & $\%$ & $\%$ & $\%$ & $\%$ & $\%$ & $\%$ & $\%$ \\
\hline $\begin{array}{l}\text { Por favor responda a cada pregunta usando una escala de } 1 \text { a } 7(1= \\
\text { totalmente en desacuerdo, } 4=\text { neutro, } 7=\text { totalmente de acuerdo) }\end{array}$ & 1 & 2 & 3 & 4 & 5 & 6 & 7 \\
\hline $\begin{array}{l}\text { Utilizamos tecnologías digitales (por ejemplo, analytics, social media, } \\
\text { dispositivos móviles, HbbTV, Big Data, Blockchain, Inteligencia artificial } \\
\text { e Internet de las cosas) para comprender mejor a nuestros públicos. }\end{array}$ & 5,6 & 4 & 4,8 & 10,4 & 21,6 & 29,6 & 24 \\
\hline $\begin{array}{l}\text { Utilizamos canales digitales (por ejemplo, social media, realidad au- } \\
\text { mentada, realidad virtual o realidad mixta y dispositivos móviles) para } \\
\text { dar a conocer nuestra organización y nuestros productos y/o servicios. }\end{array}$ & 3,2 & 1,6 & 2,4 & 9,6 & 16 & 28,8 & 38,4 \\
\hline $\begin{array}{l}\text { Utilizamos canales digitales para proporcionar servicio de atención } \\
\text { a nuestros públicos. }\end{array}$ & 1,6 & 0,8 & 0,8 & 6,4 & 21,6 & 29,6 & 39,2 \\
\hline Tenemos una visión integral de la comunicación digital. & 3,2 & 0,8 & 4,8 & 6,4 & 24,8 & 27,2 & 32,8 \\
\hline $\begin{array}{l}\text { Estamos usando análisis (métricas de reputación online y/o marca- } \\
\text { dores online) para poder tomar mejores decisiones. }\end{array}$ & 6,4 & 2,4 & 3,2 & 9,6 & 17,6 & 35,2 & 25,6 \\
\hline $\begin{array}{l}\text { Utilizamos las tecnologías digitales para aumentar el rendimiento y } \\
\text { el valor agregado de nuestros planes de comunicación. }\end{array}$ & 3,2 & 0 & 1,6 & 14,4 & 24 & 28,8 & 28 \\
\hline $\begin{array}{l}\text { Los procesos principales del departamento de comunicación están } \\
\text { automatizados mediante diferentes plataformas digitales: inves- } \\
\text { tigación, diseño, respuestas, almacenamiento de datos, envíos, } \\
\text { medición, etc. }\end{array}$ & 4 & 7,2 & 4,8 & 24 & 18,4 & 28 & 13,6 \\
\hline $\begin{array}{l}\text { Tenemos los contenidos adaptados al entorno digital (visual, inte- } \\
\text { ractivo, diseño participativo, colaborativo, personalizado, adecuada } \\
\text { frecuencia de actualización, etc.). }\end{array}$ & 2,4 & 3,2 & 1,6 & 12,8 & 14,4 & 39,2 & 26,4 \\
\hline $\begin{array}{l}\text { La formación del departamento de comunicación en comunicación } \\
\text { digital es elevada. }\end{array}$ & 3,2 & 4,8 & 3,2 & 12 & 22,4 & 30,4 & 24 \\
\hline $\begin{array}{l}\text { Estamos incorporando nuevos perfiles especializados en ámbitos } \\
\text { digitales. }\end{array}$ & 4,8 & 6,4 & 4 & 10,4 & 12,8 & 28 & 33,6 \\
\hline Medias & 3,76 & 3,12 & 3,12 & 11,6 & 19,36 & 30,48 & 28,56 \\
\hline
\end{tabular}

Fuente: elaboración propia 
En general, en esta segunda sección se observa que las evaluaciones se reparten en un volumen considerable y, en muchas ocasiones ecuánime, entre las puntuaciones 5, 6 y 7. Las medias obtenidas de cada puntuación otorgada, el 19,36\%, el 30,48\% y el 28,56\% también evidencian este volumen.

Las tres cuartas partes, el $75,2 \%$ de estos departamentos participantes, revelan que utilizan algún tipo de tecnología digital para comprender mejor a sus públicos, esto es, que estudian a sus públicos clave mediante algún tipo de tecnología para alinearse mejor con sus expectativas. La otra cuarta parte se la reparte el $14,4 \%$ de estas organizaciones, quienes dicen no disponer de ella y el 10,4\% para las que no es destacable.

El 83,2\% dicen usar diferentes canales digitales para dar a conocer la organización y sus productos o servicios, únicamente el 7,2\% de estas organizaciones se sitúan en la zona que evidencia su escaso o nulo uso de estos canales y para el 9,6\% tampoco es una cuestión a destacar. Casi la totalidad de la muestra de análisis, el 90,4\%, explica que aprovechan estos mismos canales para proporcionar un servicio de atención personalizada.

En cuanto a su destreza digital propiamente dicha, el 84,8\% de estas unidades funcionales manifiestan tener una visión integral de la comunicación digital, frente a un 8,5\% que no está muy conforme, y que no está muy seguro, en un $6,4 \%$ de los casos, que así se haga en su organización. Un 78,4\% dicen usar métricas, monitores o marcadores de reputación corporativa para poder tomar mejores decisiones, si bien algunas de ellas o bien no las utilizan, 12\%, o bien no las consideran una cuestión relevante en un $9,6 \%$.

Por otro lado, el 80,8\% de estas empresas explican que usan las tecnologías digitales para aumentar el rendimiento y el valor agregado de sus planes de comunicación, pero únicamente el $60 \%$ de los departamentos explorados confiesan tener automatizados todos sus procesos principales de comunicación, como el diseño, las respuestas, el almacenamiento de datos, envíos, o la medición, etc. El 16\% revela no tener atendida, en diferentes niveles, esta tarea y la respuesta del $24 \%$ restante es neutra.

En cambio, el $80 \%$ de las organizaciones piensan que la adaptación pedagógica al canal digital y la creatividad de los contenidos que se generan, entendida como el hecho de generar contenidos muy visuales e interactivos, colaborativos y personalizados, etc. es una cuestión muy lograda en su organización y solamente el 7,2\% no está de acuerdo en que la adaptación de los contenidos comerciales y corporativos al medio sea la más adecuada.

Por último, se observa que el $76,8 \%$ de los departamentos de relaciones públicas y comunicación analizados explican que la formación de su departamento en comunicación digital es elevada. Y, casi coincidiendo con esta cifra, el 74,4\% de los responsables sondeados explican que están incorporando nuevos perfiles especializados en estos ámbitos digitales.

Al final de esta sección también se obtiene una puntuación de entre 10 y 70 (Figura 3). En este apartado las organizaciones suman una puntuación media de 54,12, así pues, se observa que la capacidad digital, según el modelo de transformación digital de Westerman, Bonnet y McAfee (2012, 2014) es también importante puesto que la puntuación se sitúa entre 42 y 70.

Hay 105 organizaciones (84\%) de las 125 de la muestra de las empresas españolas de mayor facturación estudiadas que se presentan ante sus públicos con conocimiento, destreza y experiencia real en la aportación de este valor digital por parte del departamento de relaciones públicas y comunicación.

La capacidad digital de la empresa española se sitúa en la parte alta de la matriz entre los denominados fashionistas y los digital masters, tal como aparece representado en la Figura 3. 
Figura 3. Capacidades digitales del departamento de Relaciones Públicas y Comunicación

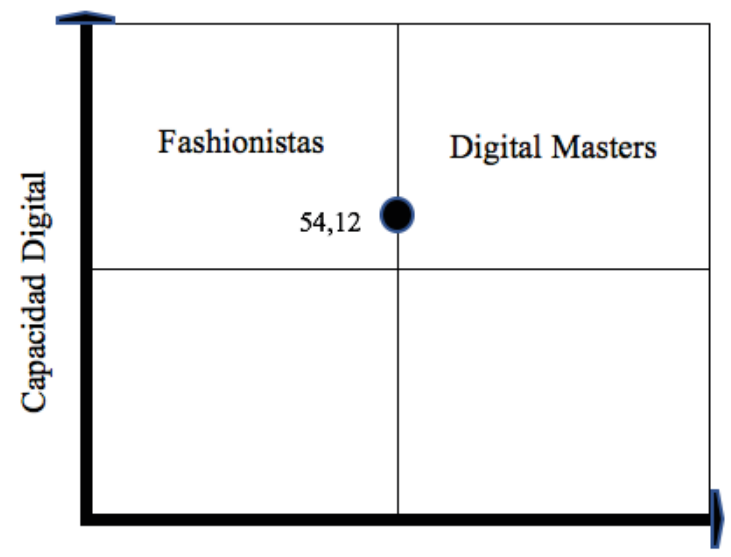

Fuente: elaboración propia.

Así pues, cuando se relacionan los dos vectores: liderazgo y capacidad digital, se observa que la muestra de los departamentos de relaciones públicas y comunicación de la industria española se sitúan en el selecto grupo de digital masters (ver Figura 4), según la matriz que resulta del modelo de Madurez Digital.

Figura 4. Madurez Digital de los departamentos de Relaciones Públicas y Comunicación de las empresas españolas de mayor facturación

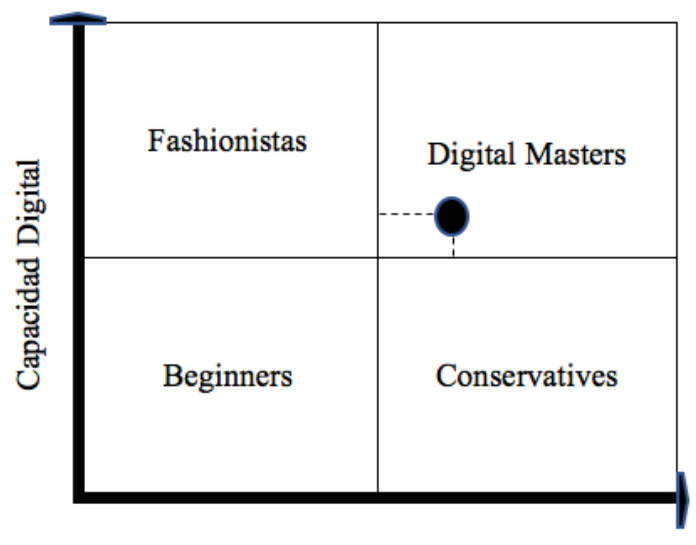

Capacidad de liderazgo

Fuente: elaboración propia.

\section{Discusión}

\subsection{Sobre las capacidades de liderazgo del departamento de Relaciones Públicas y Comunicación de las organizaciones participantes}

Como se ha indicado, la transformación digital requiere de un fuerte liderazgo para impulsar el cambio. Y, ciertamente, se observa que los departamentos de relaciones públicas y comunicación de las compañías españolas participantes en el estudio se muestran capaces ante este reto digital e indican, incluso, estar en un momento bastante avanzado de su transformación digital. Una realidad sensiblemente congruente con los optimistas resultados de los diversos informes consultados. 
La mayoría de los más altos directivos de los departamentos de comunicación tienen una visión transformadora del futuro digital. Este aspecto es esperanzador y dibuja un horizonte optimista para este desarrollo, impulso que coincide con lo que defienden a nivel global el estudio del MIT Sloan Management Review y Deloitte (Kane, Palmer, Nguyen, Kiron y Buckley, 2018) y el informe Forrester (Johnson, 2019), ya que ambos estudios afirman que más de la mitad de las empresas que empiezan un proceso de transformación digital consideran que el papel del CEO es determinante para conseguir el éxito. El Informe de Minsait 2018 revela que más de las tres cuartas partes de las direcciones generales de las grandes corporaciones españolas lideran este cambio con el ejemplo diario (Beldarrain, 2018). Esta visión, como explica la Arthur W. Page Society en la edición de prNoticias (noviembre de 2017), es una oportunidad emergente para que las empresas de cualquier lugar del mundo diseñen sistemas digitales sofisticados, a fin de gestionar el compromiso con las partes interesadas en todos los niveles, interna y externamente.

Por otro lado, sorprende advertir cómo los dircoms consultados en este estudio aseguran que las acciones de comunicación digital están coordinadas estratégicamente con el resto de operaciones de comunicación, cuando el informe del Instituto de Economía Digital explica lo contrario y discrepa al revelar que prima la táctica sobre la estrategia, y que los objetivos que se persiguen son esencialmente cortoplacistas, quedando relegados a un segundo plano los objetivos más estratégicos (Belzunce y Aguirre, 2018). Se puede interpretar y elucubrar que los máximos directivos entrevistados consideran que la transformación digital es el reto estratégico y de supervivencia más importante de su agenda y que son conscientes de la magnitud, del riesgo y de la oportunidad.

También se observa la discrepancia en torno a que los roles y las responsabilidades para gestionar la actividad digital de comunicación se diga que están claramente definidos $(79,2 \%)$, cuando otro estudio revela que, frecuentemente, se delega esta responsabilidad a un perfil junior por no disponer de un líder senior responsable del proceso (Sánchez, 2017; Belzunce y Aguirre, 2018). Se podría elucubrar, según esta segunda investigación, que un número importante de los nuevos perfiles especializados en estos ámbitos digitales que se incorporan a la organización, adoptan una responsabilidad funcional mayor que la que les correspondería de inicio, a diferencia de lo que se afirma por parte del colectivo participante en nuestro estudio.

Una cuestión que preocupa a los directivos de los departamentos de relaciones públicas y comunicación es la medición de la contribución de su labor al global de la organización (Cuenca-Fontbona, Matilla y Compte-Pujol, 2016; Cuenca-Fontbona, 2017). En este sentido, no es excepcional comprobar como tres cuartas partes de las personas entrevistadas explican que todas las acciones digitales que se emprenden se evalúan sistemáticamente mediante algún tipo de dispositivo, también tecnológico, que permita reorientar continuamente los objetivos (75\%). Estos datos son bastante coincidentes con la información publicada por la agencia de marketing de resultados Kanlli ${ }^{5}$, que explica que el $69 \%$ de las marcas españolas dice llevar a cabo una monitorización de su reputación online. Aun así, se advierte que, si el responsable de gestionar las herramientas digitales es, con frecuencia un técnico de comunicación sin experiencia en medición (Zerfass, Verčič y Charlotte, 2017), sin poder de decisión estratégica, ni visión holística de la comunicación, es posible aventurar que, muy probablemente, enfocará sus esfuerzos en medir la gestión basada simplemente en una evaluación táctica de sus acciones.

Por último, en este apartado dedicado al liderazgo se observa como falta aún cierto recorrido en el entendimiento entre el departamento de relaciones públicas y comunicación y las unidades de tecnologías de la información. Esto podría ser debido a la novedad que supone la interacción entre dos perfiles muy diferentes, que antes no colaboraban ni se coordinaban regularmente en ningún proyecto de comunicación.

\footnotetext{
${ }^{5}$ https://www.kanlli.com/
} 
En general se observa cómo se están promoviendo los cambios culturales necesarios y cómo se procuran coordinar y evaluar estratégicamente todas las acciones de comunicación digital con el resto de operaciones de comunicación, aunque, a grandes rasgos, también se observa que la transformación del departamento de comunicación tiene aún camino por recorrer. La visión y el liderazgo en la transformación digital desde la alta dirección se entiende que tiene un impacto directo en el incremento de la cuota de negocio, y que actúa como motor de crecimiento de la economía, pero vistos los resultados obtenidos, también parece que no se les ha sacado todo el partido, observaciones congruentes con el principal titular del informe del Instituto de Economía Digital: Una transformación incompleta (Belzunce y Aguirre, 2018).

\subsection{Sobre las capacidades digitales del departamento de Relaciones Públicas y Comunicación}

El parque de canales de comunicación, de monitorización y la tecnología que usan las empresas de mayor facturación de España participantes para estudiar a sus públicos clave con el objetivo de alinearse mejor a sus expectativas, y también para ser más eficaces, se observa que es significativo. También es relevante la apuesta que hace la mayoría para proporcionar un servicio de atención personalizada mediante estos medios digitales.

Sin embargo, esta realidad contrasta con la de que los profesionales consideren que su empresa tenga un grado medio de madurez digital en lo que respecta a la automatización de los procesos que permiten tomar decisiones acerca de estos canales digitales de comunicación y conocer su rendimiento. En este sentido, los resultados obtenidos son coincidentes con las palabras del CEO de Sage Iberia, Luís Pardo, quien defiende que la combinación de incorporar competencias digitales y automatizar procesos lleva a las empresas a alcanzar la evolución a la cultura corporativa digital, haciendo crecer a las compañías y haciéndolas más eficientes. Así pues, para poder invertir el tiempo en estos compromisos de carácter más estratégico, tan demandado a fin de impactar en las organizaciones en su relación con el entorno social (Gutierrez-García y Recalde, 2018) y creativo, deberá mejorarse la tarea de automatizar todos los procesos mecánicos de la comunicación.

Es imprescindible que las empresas estén convencidas de la importancia de capacitar a toda la organización en competencias y capacidades digitales. Aunque esta investigación detalla que los departamentos de relaciones públicas y comunicación explican que la formación de su departamento en comunicación digital es importante, el estudio íncipy-inesdi (2017) discrepa al detallar que existen ciertas carencias, en especial en el uso de herramientas colaborativas digitales y de trabajo en red (Sánchez, 2017). Esta formación exigiría, además, el desarrollo de competencias y capacidades como la de filtrar información, valorar y seleccionar fuentes, proteger la seguridad y privacidad de los datos, hacer un uso crítico y ético de la información y generar interacciones ricas que permitan relaciones constructivas con otras personas, etc. (Martínez-Bravo y Sádaba, Serrano, 2018).

De hecho, el cambio tecnológico seguirá aumentando, por lo que el aprendizaje de nuevas habilidades será una necesidad constante durante toda la vida profesional de los comunicadores (Sánchez, 2019). Sin embargo, el departamento de relaciones públicas y comunicación de la muestra de análisis de esta investigación se presenta con conocimiento, destreza y experiencia digital. El estudio de Roland Berges (2018) coincide y refuerza esta conclusión de nuestra investigación, pero también apunta que todavía existe un cierto recelo respecto a usos más avanzados, que únicamente son aprovechados por unas pocas empresas innovadoras. En este aspecto las organizaciones participantes se situarían muy cerca de la categorización de conservatives, es decir, de aquellas que no arriesgan en la implementación digital porque no están dispuestas a invertir los recursos económicos necesarios que ello conlleva. 
Ante el $42 \%$ de las grandes empresas que no cuentan con trabajadores especializados en digitalización, según el informe Entrepreneurship at Glance elaborado por la OCDE (2018), resulta alentador comprobar en este estudio como las organizaciones participantes, aunque en diferente medida, están incorporando a personas funcionalmente competentes en el entorno del ágora digital. Esta predisposición es probable que se vea reflejada en el futuro en unos mejores resultados desde diversos ángulos, el estratégico -es decir, en una mejor visión integradora de la comunicación eficaz-, y el creativo -de una mejorada pedagogía de los contenidos adaptados al entorno digital-, para cuya verificación serán necesarias futuras investigaciones.

\section{Conclusiones}

La transformación digital es reconocida por la muestra de análisis, en general, como de vital importancia para el éxito de la organización. Los departamentos de relaciones públicas y comunicación de la industria española de mayor facturación estudiados se sitúan en el selecto grupo de digital masters, según la matriz que resulta del modelo de Madurez Digital (IMD), mostrando unos niveles de liderazgo y de capacitación digital importantes, una posición que confirmaría la hipótesis que planteaba esta investigación conforme la digitalización de los departamentos de relaciones públicas y comunicación de las organizaciones españolas es elevada.

La industria española participante es consciente y está predispuesta a usar la tecnología e invertir recursos en ella para ser más competitiva. Los máximos responsables del área de comunicación parecen haber tomado el control de esta metamorfosis con el principal objetivo de ser más competitivos, aunque, vistos los resultados de esta investigación, el reto sigue situándose en una zona de work in progress. Se observa principalmente la tendencia respecto a que la incorporación de nuevo talento, el entendimiento con los responsables de tecnologías de la información y la automatización de procesos plantean los mayores desafíos para los esfuerzos de transformación digital en España.

En próximas investigaciones se plantea duplicar este mismo estudio, si bien aplicado a las "pymes" españolas, con la finalidad de comparar los resultados y colegir si los resultados obtenidos de ambos estudios presentan similitudes o divergencias y, por consiguiente, poder reflexionar más ampliamente en torno a la relación madurez digital/competitividad empresarial propuesta por el estudio original estadounidense (2012).

La investigación que ha conducido a estos resultados ha sido realizada mediante fondos procedentes de la Obra Social-la Caixa.

\section{Bibliografía}

Aced, C. (2018). Relaciones públicas 2.0. Cómo gestionar la comunicación corporativa en el entorno digital. Barcelona: UOC.

Aced, C., Arqués, N., Benítez, M., Llodrà, B. y Sanagustín, E. (2009). Visibilidad. Cómo gestionar la reputación en Internet. Barcelona: Gestión 2000.

Alexander, T., Stefanova, V., y Zahidi, S. (2018). The Future of Jobs Report 2018. Cologny/

Geneva (Switzerland): World Economic Forum. http://www3.weforum.org/docs/WEF_Future_of_ Jobs_2018.pdf

Baller, S., Dutta, S. y Lanvin, B. (2016). The Global Information Technology Report 2016.

World Economic Forum (2016) The Global Information Technology Report 2016. http://www3. weforum.org/docs/GITR2016/WEF_GITR_Full_Report.pdf 
Beldarrain, C. (coord.). (2018). Informe Minsait sobre la Madurez Digital en España. Madrid: Ascendant-Minsait. An Indra company. https://www.minsait.com/es/actualidad/insights/informeminsait-sobre-la-madurez-digital-en-espana

Belzunce, M., Toledo, R. y Fernández, C. (2018). La trasformación digital en 2018. Más allá de la tecnología. TD-Ebook. Madrid: Cibernos. https://servicio.cibernos.com/ebook-guia-rapidatransformacion-digital

Belzunce, M. y Aguirre, D. (2018). Una transformación digital aún incompleta. Un reto para los CEO y CIO. ler Estudio sobre la realidad de la transformación digital. Dando voz a los CIO, CDO y CTO de empresas españolas. Madrid: ICEMD-ESIC. http://cort.as/-GMKx

Berges, R. (2018). España 4.0: el reto de la transformación digital de la economía. Madrid: Roland Berges. https://w5.siemens.com/spain/web/es/estudiodigitalizacion/Documents/Estudio_ Digitalizacion_Espana40_Siemens.pdf

Cabrera, Mª . A. y Almansa-Martínez, A. (2016). El director de Relaciones Públicas en las grandes empresas españolas, Revista Internacional de Relaciones Públicas, Núm. 11, Vol. 6, pp. 113-134.

Castillo-Esparcia A., y Smolak Lozano E. (2017). Relaciones públicas digitales. Análisis de las estrategias de comunicación de los think tanks. Obra Digital, (13), 59-80.

Cook, F. (2019). PR: TECH. The future of technology in communication. USC Annenberg Center for Public Relations. Los Angeles, CA: USC Annenberg School for Communication and Journalism. https://annenberg.usc.edu/research/center-public-relations/global-communicationsreport

Crespo, B. y Pariente, E. (2018). Barómetro sobre la madurez digital en España 2018. Madrid: Divisadero. A Merkle Company - ie Business School. https://www.divisadero.es/wp-content/ uploads/barometro-madurez-digital-espana-2018.pdf

Cuenca-Fontbona, J. (2017). Cómo hacer un plan estratégico de comunicación. Vol. III: la investigación estratégica preliminar. Barcelona: Colección Dircom, Editorial UOC (Oberta UOC Publishing, SL)

Cuenca-Fontbona, J., Matilla, K. y Compte-Pujol, M. (2016). Medición de las relaciones públicas mediante monitores, indicadores y técnicas online en una sociedad conectada: un estudio vertical. En Matilla, K. (coord.). Casos de estudio de relaciones públicas. sociedad conectada: empresas y universidades. Barcelona: Colección Dircom, Editorial UOC (Oberta UOC Publishing, SL).

DESI Report 2019. (2019). The Digital Economy \& Society Index (DESI) Digital Single Market 2018. https://ec.europa.eu/digital-single-market/en/desi

Gordon, A. (2011). Public Relations. Oxford/New York: Oxford University Press.

González-Canomanuel, L. (2019). The Changing Trend of PR Globally - An Agency Perspective. Phoenix AR: International Public Relations Network-IPRN. https://iprn.com/

González Molina, S. (2011). El Dircom en el escenario de la convergencia: las claves para una transformación. Revista Internacional de Relaciones Públicas. Núm. 2, pp. 119-137.

Gutiérrrez-García, E. y Recalde, M. (2018). "Dibujando al profesional del mañana: estudio Delphi sobre capacidades de la comunicación estratégica en España”. Anàlisi: Quaderns de Comunicació i Cultura, 59, 139-156. https://www.raco.cat/index.php/Analisi/article/download/347228/438410 
Huawei (2018). Tap into New Growth with Intelligent Connectivity Mapping your Transformation into a Digital Economy with GCI 2018. https://www.huawei.com/minisite/gci/assets/files/ gci_2018_whitepaper_en.pdf?v=20180914

I-Scoop.eu (2018). Digital transformation: online guide to digital business transformation. Belgium: i-scoop. https://www.i-scoop.eu/digital-transformation/

IDC Research España (2019). Indicadores Digitales en la empresa española. Madrid: IDC Research España. https://idcspain.com/COMMONS/ATTACHMENTS/Indicadores_Digitales_ Resumen_Ejecutivo.pdf

Johnson, C. (2019) Predictions 2019: Transformation Goes Pragmatic. Cambridge (USA): Forrester Research. https://go.forrester.com/blogs/predictions-2019-transformation-goes-pragmatic/

Kane, G.C., Palmer, D., Nguyen, A., Kiron, D. y Buckley, N. (2018). Coming of Age Digitally. Massachusetts (USA): MIT Sloan Management Review and Deloitte. https://shop.sloanreview.mit. edu/store/coming-of-age-digitally

Kelly, K. (1997 enero, 9). New Rules for the New Economy. New York, NY: Condé Nast Publications. Wired. https://www.wired.com/1997/09/newrules/

Lee, A. (2013). Welcome to The Unicorn Club: Learning From Billion-Dollar Startups. New York, NY, Sunnyvale, CA: Verizon Communication. https://techcrunch.com/2013/11/02/welcometo-the-unicorn-club/?guccounter=1

Len-Ríos, M. E. y Cameron, G. T. (2001). Playing by the rules: Relationships with online users Rates (Rules-Appropriate Testing Evaluation Scale) and implications for e-commerce and portal Web sites. [Report]. Gainesville, FL: The Institute for Public Relations.

Linares, J. (dir.) (2018). Revolución digital. Impacto de las nuevas tecnologías en el directivo. Madrid: Fundación CEDE.

Lorenzo Ochoa, O. (2016). Modelos de madurez digital. ¿En qué consisten y qué podemos aprender de ellos? Deusto: Boletín de Estudios Económicos. Vol. 71, núm. 219, pp. 573-590. https://www.researchgate.net/publication/313798566_Modelos_de_Madurez_Digital_en_que_ consisten_y_que_podemos_aprender_de_ellos

Maciejewski, M. y Gouardères, F. (2018). Una Agenda Digital para Europa. Bruxelles: Parlamento Europeo. https://techcrunch.com/2013/11/02/welcome-to-the-unicorn-club/?guccounter=1

Martínez-Bravo, M. C., Sádaba, C. y Serrano, J. (2018). Desarrollo de competencias digitales en comunidades virtuales: un análisis de «Scolartic». Prisma Social, núm. 20, pp. 129 a 159. http://isdfundacion.org/2018/06/20/mejora-la-competencia-digital-con-la-participacion-encomunidades-virtuales/

Matilla, K., Miranda, T., Compte-Pujol, M. y Oliveira, A. (2018). Divergencias en la Denominación Funcional de los Departamentos de Comunicación en España. En Relaciones Públicas y contemporaneidad 4.0. Redmarka. Revista de Marketing Aplicado, vol. 01 (022), 129-156.

OCDE (2018). Entrepreneurship at a Glance. Highlights 2018. París: Organización para la Cooperación y el Desarrollo Económicos-OCDE. https://www.oecd.org/sdd/business-stats/EAG2018-Highlights.pdf

Redacción PrNoticias (noviembre de 2017). El director de Comunicación, líder en la transformación digital de la compañía. Madrid: Grupo PrNoticias. http://cort.as/-GJKx 
Rodríguez, P. y Castillo, A. (2017). Sociedad Digital en España. Madrid: Ariel y Fundación Telefónica, en colaboración con Editorial Planeta. https://www.fundaciontelefonica.com/arte cultura/publicaciones-listado/pagina-item-publicaciones/itempubli/625/

Sánchez, J. y Fernández, M. (2019). TOP 25 Profesiones Digitales 2019. Las nuevas profesiones tecnológicas y disruptivas. Barcelona: inesdi digital business school-íncipy-indigital advantage. http://cort.as/-GJMH

Sánchez, J. (2017). Índice de Madurez Digital de las Empresas. Primer estudio en España. Barcelona: íncipy- inesdi digital business school. https://incipy.com/ebooks/transformaciondigital.pdf

Sánchez, J. (2015). Transformación e innovación digital. Cómo innovar en nuevos modelos de negocio y en nuevas experiencias digitales de tus clientes. Barcelona: íncipy- inesdi digital business school. http://cort.as/-GJMH

Sato, C. y Wang, R. (2019). Constellation Research 2018 Digital Transformation Study. Digital Transformation Efforts Yield Positive ROI. Silicon Valley (USA): Constellation Research Inc. https://www.constellationr.com/research/constellation-research-2017-digital-transformation-study

Schallmo, D. y Williams, C. (2017). Digital Transformation of Business Models - Best Practice, Enablers and Roadmap. International Journal of Innovation Management, 21(8): 1740014.

Smith, J. y Davies, R. (2019). 2019 The Year of Digital Decisions. London: The Economist Intelligence Unit Limited. http://assets1.dxc.technology/digital_transformation/downloads/ Digital_Decisions_Survey_Report.pdf

Teichert, R. (2019). Digital Transformation Maturity: A Systematic Review of Literature. Acta Universitatis Agriculturae et Silviculturae Mendelianae Brunensis, 67(6): 1673-1687.

Westerman, G., Bonnet, D. \& McAfee, A. (2014). Leading Digital: Turning Technology into Business Transformation. Boston, Massachusetts (USA): Harvard Business Review Press.

Westerman, G., Bonnet, D. y McAfee, A. (2012): The Advantages of Digital Maturity. Cambridge (UK): MIT Sloan Management Review. November 20. https://sloanreview.mit.edu/article/theadvantages-of-digital-maturity/

Wilcox, D. L., Cameron, G. T. y Reber B. H. (2015). Public Relations. Strategies and Tactics. Global Edition. Harlow (UK): Pearson Education Limited.

Zerfass, A., Verčič, D. y Charlotte, S. (2017). Communication evaluation and measurement Skills, practices and utilization in European organizations. Corporate Communications: An International Journal, Vol. 22, Iss. 1, pp. 2-18. http://dx.doi.org/10.1108/CCIJ-08-2016-0056.

Zerfass, A., Verhoeven, P., Moreno, A., Tench, R. y Verčič, D. (2016). European Communication Monitor 2016. Exploring trends in big data, stakeholder engagement and strategic communication. Results of a survey in 43 Countries. Brussels: EACD/EUPRERA, Quadriga Media Berlin.

Zerfass, A., Tench, R., Verčič, D., Verhoeven, P. y Moreno, A. (2014). European Communication Monitor 2014. Excellence in Strategic Communication - Key Issues, Leadership, Gender and Mobile Media. Results of a Survey in 42 Countries. Brussels: EACD/EUPRERA, Helios Media. 